\title{
Pharmacokinetics and safety of calcium L-threonate in healthy volunteers after single and multiple oral administrations
}

\author{
Hong-yun WANG, Pei HU, Ji JIANG* \\ Clinical Pharmacology Research Center, Peking Union Medical College Hospital, Chinese Academy of Medical Science and Peking \\ Union Medical College, Beijing 100730, China
}

\begin{abstract}
Aim: To evaluate the pharmacokinetics of $L$-threonate after single or multiple oral administrations and its safety profile in healthy Chinese volunteers.

Methods: This was an open-label, single- and multiple-dose study. The subjects were assigned to receive a single dose, 675, 2025, or $4050 \mathrm{mg}$, of calcium L-threonate $(n=12)$ or repeated doses of $2025 \mathrm{mg}$ twice daily for $4 \mathrm{~d}(n=12)$. Serial plasma and urine samples were analyzed with HPLC-MS/MS. Pharmacokinetic parameters of L-threonate were calculated using non-compartmental analysis with WinNonlin software.

Results: In the single dose group, $C_{\max }$ reached at $2.0 \mathrm{~h}$ and the mean $t_{1 / 2}$ was approximately $2.5 \mathrm{~h}$. Area under curve (AUC) and $C_{\max }$ increased with dose escalation, but dose proportionality was not observed over the range of 675 to $4050 \mathrm{mg}$. AUC and $C_{\text {max }}$ in the fasted subjects were lower compared with those in the non-fasted subjects. Cumulative urinary excretion of $L$-threonate over $24 \mathrm{~h}$ represented $5.9 \%$ of the administered dose with a mean $\mathrm{Cl} / \mathrm{r}$ of $0.8 \mathrm{~L} / \mathrm{h}$. In the multiple-dose study, no accumulation appeared upon repeated doses of $2025 \mathrm{mg}$ twice daily for $4 \mathrm{~d}$. There were no serious adverse events that occurred during this study.

Conclusion: Calcium $L$-threonate was well tolerated in healthy Chinese subjects, with no pattern of dose-related adverse events.

Plasma exposure increased with dose escalation, but linear pharmacokinetics were not observed over the studied doses. L-threonate was absorbed rapidly, and its absorption was enhanced by food intake. No systemic accumulation appeared after repeated administrations.
\end{abstract}

Keywords: osteoporosis; calcium L-threonate; L-threonic acid; pharmacokinetics; safety; Chinese; open-label, single dose; multiple dose

Acta Pharmacologica Sinica (2011) 32: 1555-1560; doi: 10.1038/aps.2011.138; published online 10 Oct 2011

\section{Introduction}

Osteoporosis is a bone disease that leads to an increased risk of fracture. Due to its significant prevalence worldwide, osteoporosis is now considered a serious public health concern. In 1992, there were 1.6 million people worldwide suffering from this disease $\mathrm{e}^{[1]}$, and this number is estimated to reach 6 million by $2050^{[2]}$. Aging of populations worldwide is responsible for the major increase in the incidence of osteoporosis ${ }^{[3]}$. Osteoporosis can be treated with lifestyle changes and medications. Typical medications include bisphosphonates, estrogen analogs, raloxifene, calcium salts and sodium fluoride. These medications are classified as antiresorptive or bone anabolic agents. Antiresorptive agents reduce bone resorption, whereas bone anabolic agents build bone, rather than inhibit

\footnotetext{
* To whom correspondence should be addressed.

E-mail pk.frosh@gmail.com

Received 2011-05-26 Accepted 2011-08-17
}

resorption ${ }^{[4]}$.

$L$-Threonic acid is an active metabolite of vitamin $C^{[5-8]}$. It has been reported that $L$-threonic acid exhibits significant stimulatory action on vitamin $\mathrm{C}$ uptake and prolongs the retention of vitamin $C$ in human T-lymphoma cells ${ }^{[9,10]}$. It is also well known that vitamin $C$ is a marker for osteoblast formation and has been shown to stimulate procollagen and enhance collagen synthesis ${ }^{[11-14]}$. Therefore, $L$-threonic acid may play a role in the mineralization process through its positive action on vitamin C. This hypothesis was confirmed in 1999 by Rowe $\mathrm{DJ}^{[15]}$. It was reported that in vitro treatment with ascorbatecontaining vitamin $\mathrm{C}$ metabolites enhanced the formation of the mineralized nodules and collagenous proteins and that $L$-threonate was one of the metabolites that was found to influence the mineralization process ${ }^{[15]}$. Recently, a preclinical study was performed to investigate the effect of $L$-threonate on bone resorption of rabbit osteoclasts ${ }^{[16]}$. This study contained a total of six culture groups, including one control group and 
five groups treated with drugs (calcium $L$-threonate, sodium $L$-threonate, alendronate, $17 \beta$-estradiol and calcium gluconate). The levels of type I collagen C-telopeptide $\left(\mathrm{CT}_{\mathrm{x}}\right)$ and bone slice resorptive area were measured. This study found that $L$-threonate, especially calcium $L$-threonate, inhibited the bone resorption of osteoclasts in vitro; however, the reductive effects on the $\mathrm{CT}_{x}$ level and resorptive area were not as significant as alendronate and $17 \beta$-estradiol at the same concentration.

Calcium $L$-threonate $((2 R, 3 S)-2,3,4$-trihydroxy butyric acid calcium) (Figure 1) is a novel drug developed for the treatment of osteoporosis and as a calcium supplement. Phase I clinical trials of calcium L-threonate, including tolerance, pharmacokinetics and calcium absorption evaluation, were performed in Peking Union Medical College Hospital. In this paper, the pharmacokinetics of $L$-threonate after single or multiple oral administrations and its safety profile in healthy Chinese volunteers are presented.

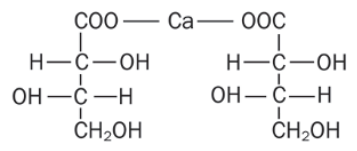

Figure 1. Calcium L-threonate, (2R,3S)-2,3,4-trihydroxy butyric acid calcium.

\section{Materials and methods}

\section{Drugs, chemical standards and reagents}

Calcium L-threonate tablets $(675 \mathrm{mg} /$ tablet $)$ and chemical standards of $L$-threonic acid (purity $99.8 \%$ ) were provided by JuNeng Pharmaceutical Co (Beijing, China). HPLC-grade acetonitrile and acetic acid were purchased from ThermoFisher (New Jersey, USA). Other reagents utilized were all of analytical grade, and distilled water was produced by a Milli$\mathrm{Q}$ water purification system (Millipore, Bedford, USA).

\section{Subjects}

This study was conducted in accordance with Good Clinical Practice regulations, the ethical principles stated in the Declaration of Helsinki, and other applicable regulatory requirements. Approval of the study was obtained from the Ethical Committee of the Peking Union Medical College Hospital (Beijing, China) prior to subject enrollment. All of the subjects provided written informed consent after the nature of the study was fully explained.

The subjects were enrolled based on the following inclusion criteria: men and women of Han Chinese ethnicity between 19 and 40 years of age with a body mass index of 19 to $25 \mathrm{~kg} / \mathrm{m}^{2}$; and no evidence of clinically significant abnormalities in cardiac, hepatic, renal, pulmonary, neurological, gastrointestinal, hematological or psychiatric function, as determined by medical history, physical examination and laboratory screens. Pregnant and lactating women were excluded from the study. Female subjects were required to be surgically incapable of pregnancy or to practice effective double-barrier birth control methods. Exclusion criteria included a history of a clinically important disease that, in the opinion of the investigator, might place the subject at risk; a history of allergy to drugs; participation in a clinical study during the preceding 4 weeks; blood donation within a period of 4 weeks prior to study drug administration; positive test results for $\mathrm{HBV}$, hepatitis $\mathrm{C}$ virus (HCV), or human immunodeficiency virus (HIV); positive test results for drugs of abuse; or a history of alcohol abuse. Additionally, all of the enrolled subjects were required to avoid vitamin $\mathrm{C}$ medications 1 month prior to and during the study.

\section{Study design}

This was a phase I, open-label, single and multiple-dose study. In the single-dose study, a randomized, 4-way crossover design was used, and the washout period between dosing was 1 week. Twelve male Chinese subjects were randomized into 4 treatment groups in which they received a single oral dose of 675,2025 , or $4050 \mathrm{mg}$ calcium $L$-threonate after an overnight fasting or $2025 \mathrm{mg}$ within $30 \mathrm{~min}$ after consuming breakfast. A standard meal was provided at 4 and $10 \mathrm{~h}$ after dosing. Serial blood samples ( $3 \mathrm{~mL}$ at each time point) were collected in heparinized vacutainers at the following time points: 0 (predose), 0.5, 1, 1.5, 2, 2.5, 3, 3.5, 4, 6, 8, 12, 24, and $48 \mathrm{~h}$. Fractionated urine samples were collected at the following time intervals: 0 (pre-dose), $0-3,3-6,6-9,9-12$, and $12-24 \mathrm{~h}$. In the multiple-dose study, 14 healthy Chinese subjects $(50 \%$ male and $50 \%$ female) received an oral dose of $2025 \mathrm{mg}$ after overnight fasting on the morning of $\mathrm{d} 1$ and $\mathrm{d} 7$, and serial blood samples ( $3 \mathrm{~mL}$ at each time point) were drawn at the following time points: 0 (pre-dose), 0.5, 1, 1.5, 2, 2.5, 3, 3.5, 4, 6, 8, 12, 24, and $48 \mathrm{~h}$. From d 3 to $\mathrm{d} 6$, the subjects received an oral dose of $2025 \mathrm{mg}$ twice daily with a dose interval of $12 \mathrm{~h}$. Blood samples prior to dosing $(0 \mathrm{~h})$ were collected in the morning for trough plasma level monitoring. During the study, blood samples were separated by centrifugation at $2500 \times g$ for $10 \mathrm{~min}$, and plasma was collected. The volume of urine samples at each time interval was recorded, and $10 \mathrm{~mL}$ was collected. All of the plasma and urine samples were stored at $-30^{\circ} \mathrm{C}$ until analysis.

All of the subjects were hospitalized in the phase I unit ward of Peking Union Medical College Hospital during the study. The subjects were required to refrain from smoking, alcohol and caffeinated beverages. Strenuous exercise was not allowed on each dosing day. Medications (including vitamins, herbal supplements and traditional Chinese medicines) were not permitted, although paracetamol (acetaminophen) was allowed as a mild analgesic.

\section{Plasma and urine sample analysis}

The concentrations of $L$-threonate in plasma and urine samples were determined using the high-performance liquid chromatography-tandem mass spectrometry (HPLC-MS/ MS) method developed in our lab ${ }^{[17]}$. Briefly, $0.1 \mathrm{~mL}$ volume of plasma sample was vortexed with $0.3 \mathrm{~mL}$ of methanol for $3 \mathrm{~min}$. After centrifugation at $10000 \times \mathrm{g}$ for $5 \mathrm{~min}, 0.2 \mathrm{~mL}$ of 
the supernatant was collected and diluted with $1.5 \mathrm{~mL}$ of water; $20 \mu \mathrm{L}$ of the sample was injected for analysis. Each $0.1 \mathrm{~mL}$ volume of urine was diluted with $2 \mathrm{~mL}$ of water and vortexed for $1 \mathrm{~min}$, and then $20 \mu \mathrm{L}$ of the sample was injected for analysis. The analyte was separated on an YMC J'Sphere $\mathrm{C}_{18}$ column (50 mm×4.6 mm; id, $3.5 \mu \mathrm{m}$ )(Waters, MA; USA) with methanol-acetonitrile-10 $\mathrm{mmol} / \mathrm{L}$ ammonium acetate $(20: 5: 75, v / v)$ as the mobile phase and at a flow rate of $0.2 \mathrm{~mL} /$ min. Detection was performed on a Quattro Ultima triplequadruple mass spectrometer (Waters, MA, USA) equipped with an electrospray source (ESI) operated in the negative ionization mode. Multiple reactions monitoring (MRM) was used to monitor $L$-threonate by the ion transition of $m / z 134.5 \rightarrow 74.7$. The linear calibration curves of $L$-threonate in plasma and urine were obtained over the concentration range of 0.25 to 50 $\mathrm{mg} / \mathrm{L}$ and 2.5 to $500 \mathrm{mg} / \mathrm{L}$. Low-, medium-, and high-quality control samples (plasma: $0.75,12.5,40 \mathrm{mg} / \mathrm{L}$; urine: $7.5,125$, $400 \mathrm{mg} / \mathrm{L}$ ) were analyzed with the study samples to ensure the quality of analysis.

\section{Pharmacokinetic and statistical analysis}

Pharmacokinetic analysis was performed with WinNonlin software (Pharsight 4.0.1; NC, USA) utilizing non-compartmental analysis. The maximum plasma drug concentration $\left(C_{\max }\right)$ and time to $C_{\max }\left(t_{\max }\right)$ were directly obtained from the plasma concentration-time curves. The terminal-phase elimination half-life $\left(t_{1 / 2}\right)$ was calculated as $0.693 / \lambda_{z}$, where $\lambda_{z}$ was the slope of the apparent elimination phase of the natural logarithmic (ln) transformation of the plasma concentration-time curve, which was estimated using liner regression. The area under the plasma concentration-time curve from time zero to $t\left(\mathrm{AUC}_{0-\mathrm{t}}\right)$, where $t$ is the time of last measurable sample, was calculated according to the linear trapezoidal rule. The AUC from time zero to infinity $\left(\mathrm{AUC}_{0-\infty}\right)$ was estimated as $\mathrm{AUC}_{0-\mathrm{t}}+C_{\mathrm{t}} / \lambda \mathrm{z}$, where $C_{\mathrm{t}}$ was the plasma concentration of the last measurable sample. Apparent total clearance $(\mathrm{Cl} / \mathrm{F})$ was calculated as Dose/ $\mathrm{AUC}_{0-\infty}$ or Dose/ $\mathrm{AUC}_{\mathrm{ss}}$ and apparent total volume of distribution $\left(V_{z} / F\right)$ as calculated as $C L / \lambda_{z}$. Renal clearance was estimated as $\mathrm{Au}_{0-\mathrm{t}} / \mathrm{AUC}_{0-\mathrm{t}}$, where $\mathrm{Au}_{0-\mathrm{t}}$ was the cumulative amount of drug excreted in urine from time zero to $t(24 \mathrm{~h})$. Attainment of steady state by $\mathrm{d} 7$ was evaluated by regressing the natural logarithmic (ln) transformation of trough concentrations on d 3,4,5, and 6 over time. Steadystate was attained if the slope was not statistically different from zero. The steady-state AUCs $\left(\mathrm{AUC}_{\mathrm{ss}}\right)$ over the dosing interval $(\tau=12 \mathrm{~h})$ and $C_{\mathrm{avg}}\left(\mathrm{AUC}_{\mathrm{ss} / \tau}\right)$ were calculated. The degree of fluctuation (DF\%) was calculated as $\left(C_{\max }-C_{\min }\right) /$ $C_{\text {avg }} \times 100 \%$. Accumulation ratios were defined as the steadystate $\mathrm{AUC}_{0-\tau}$ to the single-dose $\mathrm{AUC}_{0-\tau}$ ratio or the steady state $C_{\max }$ to the single-dose $C_{\max }$ ratio, namely, $\mathrm{R}_{\mathrm{AUC}}=A \mathrm{AUC}_{0-\tau}$ (steady state)/ $A U C_{0-\tau}$ (single-dose) $(\tau=12 \mathrm{~h})$ and $R_{C_{\max }}=C_{\max }$ (steady state) $/ C_{\max }$ (single-dose), respectively.

Statistical analysis was performed using SPSS software (version 11.0; SPSS Inc; Chicago, IL, USA). The results were expressed as the mean \pm SD. The linearity of plasma $L$-threonate pharmacokinetics within the 675 to $4050 \mathrm{mg}$ dose range was assessed with respect to pharmacokinetic parameters underlying drug exposure, $i e, \mathrm{AUC}$ and $C_{\max }$. After logtransformation, $90 \%$ confidence intervals $\left(\mathrm{CI}_{\mathrm{s}}\right)$ for the ratios of dose-normalized, geometric mean values of AUC and $C_{\max }$ were calculated using mixed-effects models. The approach was developed by Smith et al on the basis of the criteria for the assessment of bioequivalence ${ }^{[18]}$. Dose proportionality is declared if the CI for the dose ratio is contained within the limits, $80 \%-125 \%$ for AUC and $70 \%-143 \%$ for $C_{\max }$. Based on a $2025 \mathrm{mg}$ dose level, the food intake effect on the pharmacokinetics of $L$-threonate was assessed between the fasted and fed subjects using a paired- $t$ test, where $P \leq 0.05$ was considered statistically significant.

\section{Safety evaluation}

Safety and tolerability were evaluated through adverse events reported by the investigators and subjects and based on clinical laboratory measurements (blood chemistry, hematology, urinalysis, and liver functions), 12-lead ECG, physical examination, and vital signs. Adverse events were assessed by the investigators with regard to severity (mild, moderate, severe or life-threatening) and the relationship to the study treatment (reasonably or possibly related, not reasonably or possibly related).

\section{Results}

\section{Subject demographics}

Twenty-six healthy Han Chinese volunteers were recruited and randomized into the phase I clinical study. In the singledose study, all of the subjects (12 males) completed the 4-way, crossover trial. In the multiple-dose study, 14 subjects (7 males and 7 females) were enrolled, and two subjects withdrew on d 3 due to personal reasons. There were no significant differences among the 26 subjects with regard to age, height, weight, or body mass index (Table 1).

Table 1. Subject demographics, mean $\pm S D$ (range).

\begin{tabular}{lcc}
\hline & $\begin{array}{c}\text { Single-dose } \\
(n=12)\end{array}$ & $\begin{array}{c}\text { Multiple-dose } \\
(n=14)\end{array}$ \\
\hline Age (year) & $28.2 \pm 4.8(24-38)$ & $28.1 \pm 5.3(21-37)$ \\
Sex & 12 male & 7 male, 7 female \\
Height (cm) & $169.9 \pm 4.2(162-178)$ & $166.1 \pm 4.5(160-174)$ \\
Weight $(\mathrm{kg})$ & $64.6 \pm 4.1(57-71)$ & $58.6 \pm 4.1(52-64)$ \\
BMl* & $22.4 \pm 1.8(19.3-25.1)$ & $21.0 \pm 1.4(19.1-22.5)$ \\
\hline
\end{tabular}

$\mathrm{BMI}^{*}$ : body mass index

\section{Single-dose pharmacokinetics}

The mean plasma concentration-time curves in 12 healthy Chinese subjects after oral administration of 675,2025 , or $4050 \mathrm{mg}$ calcium L-threonate are shown in Figure 2, and the pharmacokinetic parameters are presented in Table 2. The results indicated that $L$-threonate was absorbed rapidly with a 


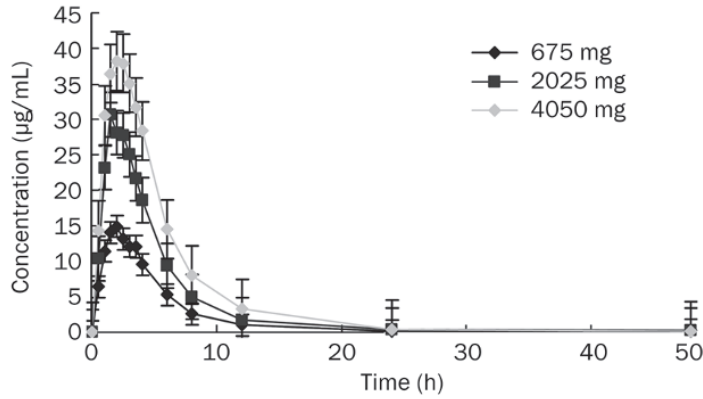

Figure 2. Mean plasma concentration-time curves in healthy Chinese subjects after single oral administration of calcium L-threonate 675, 2025, and $4050 \mathrm{mg}(n=12)$.

median $t_{\max }$ of $2.0 \mathrm{~h}$ across all of the dose cohorts. After reaching peak exposure, the plasma disposition of $L$-threonate was also rapid, having a mean $t_{1 / 2}$ of $2.5 \mathrm{~h}$, which was independent of dose. The mean oral apparent total plasma clearance $(C l / F)$ and $V_{z} / F$ were $9.8 \mathrm{~L} / \mathrm{h}$ and $28.9 \mathrm{~L}, 14.5 \mathrm{~L} / \mathrm{h}$, and $53.6 \mathrm{~L}$, and $21.3 \mathrm{~L} / \mathrm{h}$ and $76.5 \mathrm{~L}$ for the 675,2025 , and $4050 \mathrm{mg}$ dose cohorts, respectively. Over the dose range of 675 to $4050 \mathrm{mg}$, the mean $C_{\max }$ increased from 15.5 to $42.8 \mathrm{mg} / \mathrm{L}$ and $\mathrm{AUC}_{0-\infty}$ increased from 77.7 to $220.9 \mathrm{~h} \cdot \mathrm{mg} / \mathrm{L}$; however, the $90 \%$ CI for the ratio of dose-normalized, geometric mean values of $C_{\max }$ and AUC indicated that there was no apparent dose proportionality over the range of $675 \mathrm{mg}$ to $4050 \mathrm{mg}$ (Table 3).

The effect of food intake on the pharmacokinetics of L-threonate was studied in the $2025 \mathrm{mg}$ dose group, in which 12 subjects received calcium $L$-threonate after overnight fasting or breakfast. The results showed that $C_{\max }$ and AUC in fed subjects were increased compared to fasted subjects (Figure 3). The mean $C_{\max }$ increased from 32.3 to $39.1 \mathrm{mg} / \mathrm{L}$, and the mean $\mathrm{AUC}_{0-\infty}$ increased from 152.9 to $203.6 \mathrm{~h} \cdot \mathrm{mg} / \mathrm{L}$. There was a statistically significant difference between the fasted and fed groups using a paired $t$ test $(P<0.05)$; therefore, it was concluded that the absorption of $L$-threonate was improved by food intake.
Table 3. $90 \% \mathrm{Cl}$ for the ratio of dose-normalized, geometric mean values of $\mathrm{AUC}$ and $C_{\max }$.

\begin{tabular}{lcccc}
\hline & \multicolumn{2}{c}{$90 \% \mathrm{Cl}$} \\
& \multicolumn{2}{c}{ AUC $_{0-\mathrm{t}}$} & \multicolumn{2}{c}{$\mathrm{C}_{\max }$} \\
\hline & Mean* & $90 \% \mathrm{Cl}^{+}$ & Mean* & $90 \% \mathrm{Cl}^{+}$ \\
$675 \mathrm{mg}: 2025 \mathrm{mg}$ & 1.486 & $1.276-1.697$ & 1.441 & $1.300-1.582$ \\
$4050 \mathrm{mg}: 2025 \mathrm{mg}$ & 0.701 & $0.491-0.912$ & 0.658 & $0.517-0.800$ \\
CVw (\%) & $30.50 \%$ & $20.25 \%$ & & \\
\hline
\end{tabular}

$\mathrm{Cl}=(\mathrm{A}-\mathrm{B})(+/-) t_{-}$value *sqrt(MSE)*sqrt $(1 / \mathrm{nA}+1 / \mathrm{nB})$, where $\mathrm{A}-\mathrm{B}=$ mean difference between the treatment $A$ and $B$ under log scale, $t$ _value is the crirical values from t-distribution with degree of freedom of MSE, $C V w \%($ within-subject coefficients of variation $)=\operatorname{sqrt}(\exp (M S E)-1) * 100$, where MSE is the residual error from ANOVA).

$(*)=$ geometric mean

$(+)$ : lower limit-upper limit

(\#): within-subject coefficients of variation=sqrt $[\exp (\mathrm{MSE})-1] \times 100$, where MSE is the residual error from ANOVA

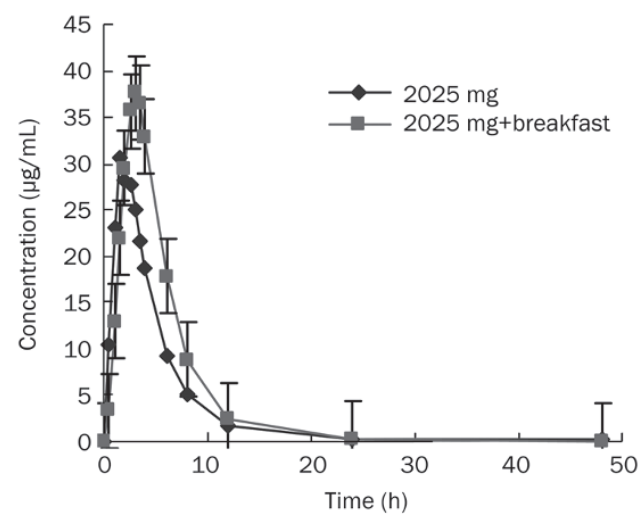

Figure 3. Mean plasma concentration-time curves in healthy Chinese subjects receiving $2025 \mathrm{mg}$ calcium L-threonate after overnight fasting or breakfast $(n=12)$.

Table 2. Pharmacokinetic parameters of $L$-threonate in healthy Chinese subjects following single oral administration (mean $\pm S D$ ).

\begin{tabular}{|c|c|c|c|c|}
\hline & \multicolumn{4}{|c|}{ Dose (mg) } \\
\hline & $675(n=12)$ & $2025(n=12)$ & 2025 (fed) $(n=12)$ & $4050(n=12)$ \\
\hline$t_{\max }($ median, $\mathrm{h})$ & $2.0 \pm 0.5$ & $2.0 \pm 0.4$ & $3.0 \pm 0.5$ & $2.0 \pm 0.7$ \\
\hline$t_{1 / 2}(\mathrm{~h})$ & $2.4 \pm 1.3$ & $2.7 \pm 0.8$ & $2.3 \pm 0.4$ & $2.7 \pm 0.6$ \\
\hline$A \cup C_{0-t}(\mathrm{mg} / \mathrm{L} \cdot \mathrm{h})$ & $76.2 \pm 30.1$ & $150.1 \pm 41.8$ & $199.7 \pm 69.5$ & $218.3 \pm 90.8$ \\
\hline $\mathrm{Cl} / \mathrm{F}(\mathrm{L} / \mathrm{h})$ & $9.8 \pm 3.3$ & $14.5 \pm 4.9$ & $11.4 \pm 4.9$ & $21.3 \pm 8.7$ \\
\hline $\mathrm{Cl} / \mathrm{r}(\mathrm{L} / \mathrm{h})$ & $1.0 \pm 0.7$ & $0.7 \pm 0.5$ & $0.7 \pm 0.3$ & $0.8 \pm 0.5$ \\
\hline $\mathrm{Au}_{0-\mathrm{t}}(\mathrm{mg})$ & $69.8 \pm 48.2$ & $84.6 \pm 75.7$ & $132.8 \pm 62.4$ & $135.5 \pm 136.6$ \\
\hline Urinary excretion rate (\%) & 10.30 & 4.20 & 6.60 & 3.30 \\
\hline
\end{tabular}


Following single administration, urine samples were collected over a period of $24 \mathrm{~h}$. Cumulative urinary excretion of $L$-threonate over the collection interval was $10.3 \%, 4.2 \%$, and $3.3 \%$ of the administered dose of 675,2025 , and $4050 \mathrm{mg}$, respectively, with a mean renal clearance of $0.8 \pm 0.5 \mathrm{~L} / \mathrm{h}$.

\section{Multiple-dose pharmacokinetics}

The mean plasma concentration-time curves of $L$-threonate in 12 healthy Chinese subjects following multiple-dose administration of $2025 \mathrm{mg}$ calcium $L$-threonate are presented in Figure 4 , and the pharmacokinetic parameters are presented in Table 4. Attainment of steady-state was achieved by d 7 because the regression slope of natural logarithmic (ln) transformation of trough concentrations on $\mathrm{d} 3,4,5$, and 6 over time was not statistically different from zero. The mean steady-state $t_{1 / 2}$ was $2.1 \pm 0.6 \mathrm{~h}$, which was comparable to the value of a singledose $(2.0 \pm 0.7 \mathrm{~h})$. The accumulation index was $1.086(90 \% \mathrm{CI}$, 0.967-1.204) based on $\mathrm{AUC}_{0-\mathrm{t}}$ and 1.102 (90\% CI 1.004-1.2) based on $C_{\max }$ indicating that there was no further accumulation of $L$-threonate after multiple administrations.

\section{Safety evaluation}

There were no serious adverse events (AEs) reported in this

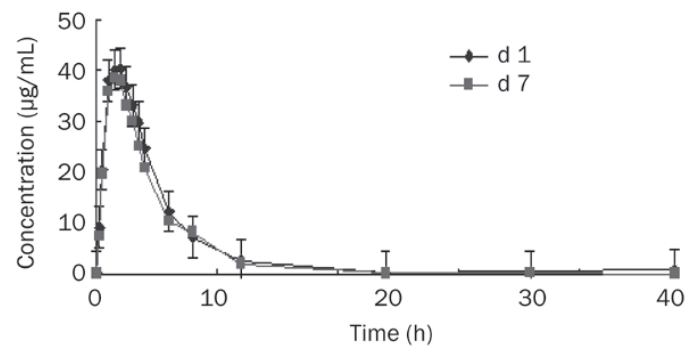

Figure 4. Mean plasma concentration-time curves in healthy Chinese subjects after multiple doses of $2025 \mathrm{mg}$ calcium $L$-threonate $(n=12)$.

Table 4. Pharmacokinetic parameters of calcium L-threonate in healthy Chinese subjects following multiple-dose of 2025 mg calcium $L$-threonate (mean $\pm S D$ ).

\begin{tabular}{lcc}
\hline & $\mathrm{d} 1(n=12)$ & $\mathrm{d} 7(\mathrm{n}=12)$ \\
\hline$t_{\max }($ median, h) & $1.6 \pm 0.6$ & $1.6 \pm 0.4$ \\
$C_{\max }(\mathrm{mg} / \mathrm{L} \cdot \mathrm{h})$ & $43.8 \pm 16.4$ & $47.7 \pm 15.3$ \\
$t_{1 / 2}(\mathrm{~h})$ & $2.1 \pm 0.6$ & $2.0 \pm 0.7$ \\
$\mathrm{AUC} \mathrm{C}_{0-\mathrm{t}}(\mathrm{mg} / \mathrm{L} \cdot \mathrm{h})$ & $204.1 \pm 88.2$ & $216.2 \pm 68.1$ \\
$\mathrm{AUC} \mathrm{C}_{0-\infty}(\mathrm{mg} / \mathrm{L} \cdot \mathrm{h})$ & $207.5 \pm 87.0$ & $220.4 \pm 67.1$ \\
$V_{z} / \mathrm{F}(\mathrm{L})$ & $34.4 \pm 16.5$ & $29.3 \pm 10.2$ \\
$C l / F(\mathrm{~L} / \mathrm{h})$ & $12.3 \pm 6.6$ & $13.6 \pm 3.62$ \\
$C_{\text {avg }}(\mathrm{mg} / \mathrm{L})$ & - & $14.6 \pm 2.66$ \\
$\mathrm{AUC} C_{\mathrm{ss}}(\mathrm{mg} / \mathrm{L} \cdot \mathrm{h})$ & - & $176.0 \pm 31.6$ \\
$\mathrm{DF}(\%)^{*}$ & - & $3.1 \pm 1.1$ \\
$\mathrm{R}_{\mathrm{C}_{\max }}$ & - & 1.102 \\
$\mathrm{R}_{\mathrm{AUC}}$ & & 1.086 \\
\hline
\end{tabular}

*: DF, degree of fluctuation. study. The major adverse event was diarrhea (1/26, 3.8\%), which was observed in one subject after administration of 2025 mg calcium $L$-threonate during the single-dose period. This AE was mild, transient, and without fever, nausea or bellyache; the subject recovered without medication or other treatment. No additional AEs were reported in this study. There were no clinically significant changes in laboratory parameters (clinical chemistry, hematology or urinalysis) or vital signs after treatment with calcium $L$-threonate. When compared to baseline values acquired in the screening period, there were no clinically significant changes in the ECG data. Therefore, calcium $L$-threonate was shown to be safe and well tolerated in healthy Chinese subjects.

\section{Discussion}

Calcium $L$-threonate is a novel drug developed for the treatment of osteoporosis. In this study, the pharmacokinetics and safety of $L$-threonate in healthy Chinese volunteers were reported for the first time. This study was divided into two parts: single- and multiple-dose studies. In the single-dose study, a 4-way, crossover design was used; 12 male Chinese subjects received 675, 2025, 2025 (after breakfast) or 4050 mg calcium $L$-threonate. It indicated that $L$-threonate was absorbed rapidly, with $C_{\max }$ being reached at a median time of $2.0 \mathrm{~h}$. Over the dose range of $675 \mathrm{mg}$ to $4050 \mathrm{mg}$, the plasma exposure of $L$-threonate increased with dose escalation; however, dose proportionality was not observed. The reason was not clear, but it could be due to the saturate absorption of $L$-threonate. After reaching peak exposure, the disposition of $L$-threonate was rapid, as $t_{1 / 2}$ ranged from 2.4 to $2.7 \mathrm{~h}$. $\mathrm{AUC}_{0-\mathrm{t}}$ contributed to over $98 \%$ of the total AUC, indicating that a sampling period of $48 \mathrm{~h}$ was adequate for assessing plasma exposure of $L$-threonate. Cumulative urinary excretion of $L$-threonate over $24 \mathrm{~h}$ represented only $5.9 \%$ of the administered dose. Because L-threonate is an acidic compound that has hydroxyl and carboxyl groups, we hypothesized that this drug might be eliminated as a conjugated form. However, no phase II metabolites were found in plasma or urine when we screened the samples using a TOF mass spectrometer (QSTAR XL, AB Sciex, USA). Therefore, we hypothesize that L-threonate may be involved in the anabolism of other endogenous substances after administration. The metabolic pathway of $L$-threonate is still being investigated in our lab.

In the multiple-dose study, there was no accumulation after repeated doses of $2025 \mathrm{mg}$ twice per day for $4 \mathrm{~d}$. The mean $t_{1 / 2}$ was similar to that of the single-dose study, indicating that the elimination rate of $L$-threonate did not change during repeated administrations. However, concerning the $2025 \mathrm{mg}$ dose group, AUC and $C_{\max }$ in the multiple-dose study were significantly higher than those of the single-dose study (fasted group), approximately $30 \%$ higher based on the $C_{\max }$ and AUC. A combined total of 24 Chinese subjects (18 male, 6 female) completed this study. Half of the subjects in the multiple-dose group were male $(n=6)$, and half were female $(n=6)$, whereas all of the subjects $(n=12)$ in the single-dose group were male. Therefore, is the difference in AUC and $C_{\max }$ between the 
single- and multiple-dose studies due to gender effect? Pharmacokinetic parameters $\left(C_{\max }\right.$ and $\left.\mathrm{AUC}_{0-\mathrm{t}}\right)$ were compared using subject, gender and period as fixed factors using a threeway ANOVA analysis. This analysis indicated that the differences among subjects and periods were significant $(P<0.01)$; however, there was no statistical difference between male and female subjects $(P>0.05)$. Therefore, the differences in plasma exposure were due to the study period and individual variation, and not gender.

The safety evaluation during the study demonstrated that calcium $L$-threonate was well tolerated in healthy Chinese volunteers over the studied dose range. The reported $\mathrm{AE}$ was diarrhea $(1 / 26,3.8 \%)$, which was possibly due to mannitol, an excipient used in the tablet, increasing the intestinal osmotic pressure. This reported $\mathrm{AE}$ was mild and transient; the subject recovered without any medication or further treatment.

In summary, this study demonstrated the single- and multiple-dose pharmacokinetics of calcium $L$-threonate and its safety profile in healthy Chinese volunteers. $L$-threonate was absorbed rapidly and exhibited dose-related plasma exposure. After reaching peak exposure, this drug was quickly cleared from the plasma, but renal excretion was not its major elimination route. The absorption of $L$-threonate was enhanced by food intake, and there was no accumulation after multiple administrations. Calcium $L$-threonate was well tolerated in healthy Chinese volunteers over the studied dose range.

\section{Acknowledgements}

This study was supported by JuNeng Pharmaceutical Co (Beijing, China). The authors thank all of the volunteers enrolled in this study. We also thank the staff of the clinical ward and bioanalytical lab of Phase I Unit in Peking Union Medical College Hospital.

\section{Author contribution}

Ji JIANG and Pei HU designed the research and revised the manuscript; Hong-yun WANG performed the research, analyzed the data, and wrote the paper.

\section{References}

1 Cooper C, Campion G, Melton $\sqcup$ 3rd. Hip fractures in the elderly: a world-wide projection. Osteoporosis Int 1992; 2: 285-9.
2 Sambrook P, Cooper C. Osteoporosis. Lancet 2006; 367: 2010-8.

3 Reginster JY, Burlet N. Osteoporosis: A still increasing prevalence. Bone 2006; 38: 4-9.

4 Mauck KF, Clarke BL. Diagnosis, screening, prevention, and treatment of osteoporosis. Mayo Clin Proc 2006; 81: 662-72.

5 Thomas M, Hughes RE. A relationship between ascorbic acid and threonic acid in guinea-pigs. Food Chem Toxicol 1983; 21: 449-52.

6 Chamberlin BA, Sweeley CC. Metabolic profiles of urinary organic acids recovered from absorbent filter paper. Clin Chem 1987; 33: 572-6.

7 Thomas M, Hughes RE. Evaluation of threonic acid toxicity in small animals. Food Chem 1985; 17: 79-83.

8 Isbell HS, Frush HL. Oxidation of $L$-ascorbic acid by hydrogen peroxide: Preparation of L-threonic acid. Carbohydr Res 1979; 72 : 301-4.

9 Fay MJ, Verlangieri AJ. Stimulatory action of calcium L-threonate on ascorbic acid uptake by a human T-lymphoma cell line. Life Sci 1991; 19: 1377-81.

10 Fay MJ, Bush MJ, Verlangieri AJ. Effect of aldonic acids on the uptake of ascorbic acid by 3T3 mouse fibroblasts and human T lymphoma cells. Gen Pharmacol 1994; 25: 1465-9.

11 Morton DJ, Barrett-Connor EL, Schneider DL. Vitamin C supplements use and bone mineral density in postmenopausal women. J Bone Miner Res 2001; 16: 135-40.

12 Hall SL, Greendale GA. The relation of dietary vitamin C intake to bone mineral density: results from the PEPI study. Calcif Tissue Int 1998; 63: 183-9.

13 Franceschi RT, Lyer BS. Relationship between collagen synthesis and expression of the osteoblast phenotype in MC3T3-E1 cells. J Bone Miner Res 1992; 7: 235-46.

14 Franceschi RT, Lyer BS, Cui Y. Effects of ascorbic acid on collagen matrix formation and osteoblast differentiation in murine MC3T3-E1 cells. J Bone Miner Res 1994; 9: 843-54.

15 Rowe DJ, Ko S, Tom XM, Silverstein SJ, Richards DW. Enhanced production of mineralized nodules and collagenous proteins in vitro by calcium ascorbate supplemented with vitamin C metabolites. J Periodontol 1999; 70: 992-9.

$16 \mathrm{He} \mathrm{JH}$, Tong NW, Li HQ, Wu J. Effects of L-threonate on bone resorption by osteoclasts in vitro. J Sichuan Univ (Med Sc Edi) 2005; 36: 225-8.

17 Wang HY, Jiang J, Hu P. Determination of $L$-threonate in human plasma and urine by high performance liquid chromatography-tandem mass spectrometry. J Chromatogr B 2006; 834: 155-62.

18 Smith BP, Vandenhende FR, DeSante KA, Farid NA, Welch PA, Callaghan JT, et al. Confidence interval criteria for assessment of dose proportionality. Pharm Res 2000; 17: 1278-83. 